\title{
Research on Automatic Positioning Algorithm of Fire Point by Video Image in Intelligent Forest
}

\author{
Gaohe $\mathrm{Li}^{1}{ }^{1,}$, Yanli Zhang ${ }^{2}$ \\ ${ }^{1}$ School of Economic Management, Xi'an Shiyou University, Xi'an, China \\ ${ }^{2}$ International Business School, Shaanxi Normal University, Xi'an, China \\ Email address: \\ gaoheli@xsyu.edu.cn (Gaohe Li), yanli_agent@126.com (Yanli Zhang) \\ ${ }^{*}$ Corresponding author
}

To cite this article:

Gaohe Li, Yanli Zhang. Research on Automatic Positioning Algorithm of Fire Point by Video Image in Intelligent Forest. International Journal of Information and Communication Sciences. Vol. 4, No. 1, 2019, pp. 18-23. doi: 10.11648/j.ijics.20190401.13

Received: April 21, 2019; Accepted: May 28, 2019; Published: June 12, 2019

\begin{abstract}
Based on the digital video monitoring system in smart forest, the automatic positioning algorithm of forest fire is studied by using camera calibration technique and spatial stereo analysis. Using the method of exhaustive search and dichotomy, the location of the fire point on the terrain profile is determined by DEM model and using the principle of stereoscopic geometry. According to the characteristics of the forest terrain changes, using translation methods of the camera optical axis in the space, the mapping relationship between the plane pixel coordinates and the spatial coordinates is established. The research simplifies the algorithm. It reduces the complexity of the algorithm, reduces the intermediate calculation link, and avoids the cumulative error of multiple calculations, and improves the calculation accuracy. In the algorithm proposed in this paper, after the test of more than 40 groups of data (due to limited space, this article only lists 24 sets of data) in two geographical locations, the straight-line distance error of the two previous calculations of the fire location is within $95 \mathrm{~m}$, and the accuracy of the rotation Angle and pitch Angle is greatly improved. The actual application shows that the localization algorithm can meet the automatic positioning of forest fire point and is an important part of intelligent forest monitoring system.
\end{abstract}

Keywords: Forest Fire, Automatic Positioning, Digital Elevation Model, Camera Calibration, Exhaustive Search Method, Dichotomy

\section{Introduction}

Around the world, about 200,000 forest fires occur annually, burning more than 6.4 million hectares. Forest fires have become one of the main reasons for the decrease of forest resources. The occurrence of forest fires is uncertain to a certain extent, and the location of the ignition point is random to a certain extent. It often occurs in remote places and is not easy to monitor. After the fire, the fire quickly, the fire spread faster, the longer the fire spread the more difficult to fight, leading to major casualties and property losses. [1-3] Recent wildfires in the United States and Australia are an example. Therefore, the timely location of fire ignition point is crucial for forest fire prevention. Satellite remote sensing, aircraft and unmanned aerial vehicles (uavs) and ground surveillance, such as manned watch, watchtowers and cloud platforms, are common methods used to locate and locate ignition points. In the air, satellite remote sensing is characterized by wide monitoring range and high positioning accuracy. Disadvantages are high cost and poor real-time performance. Aircraft and unmanned aerial vehicles (uavs) are characterized by accurate positioning. Disadvantages are also high cost, poor real-time, poor duty. On the ground, manual duty is characterized by more personnel, higher labor intensity, lower positioning accuracy, and failure to timely obtain fire site data. The automatic monitoring of watchtower is characterized by accurate positioning, low cost, and in line with the actual situation of technology development at the present stage. The technology of automatic monitoring of watchtower includes sensor, radar, infrared and digital video monitoring. Among them, the penetration rate of digital video monitoring technology is higher and the price is cheaper. [46] 
In 1958, Miller and Laflamme first put forward the Digital expression of computerized terrain element information, namely Digital Terrain Model (DTM). [7] It is a simple statistical representation of continuous ground by using a large number of selected known $\mathrm{X}, \mathrm{Y}$ and $\mathrm{Z}$ coordinate points in a coordinate system. The ground has a wide range of properties, which can refer to the distribution of elevation, population, rainfall, temperature, air pressure and so on in an area. When the ground feature of a Digital Terrain Model is elevation, it is called DEM (Digital Elevation Model). DEM uses a set of ordered numerical array to express and simulate the spatial distribution of ground elevation in discrete numbers, which can directly reflect the continuous relief of the terrain on the earth surface. DEM has been adopted by Germany's Fire-Watch, Canada's ForestWatch, France's UraFire, South Africa's Fire-Hawk, Britain's FireVu and Croatia's iForestFire. The fire location method proposed in this paper is the digital video monitoring system algorithm based on DEM model.

If single point positioning can determine the location of the fire point, double points and multiple points can definitely locate the fire point. [8-13] Therefore, this paper only describes the single-point positioning algorithm. According to the reference materials, the single point location algorithm based on the digital video monitoring system is generally tedious and of high complexity. The accuracy of some algorithms is that the latitude error is within 150 meters and the longitude error is within 120 meters. If the straight-line distance error of two points is calculated, it is more than this data. Some algorithms also have relatively large angle errors. In the algorithm proposed by the author in this paper, 10 sets of data provided by a party are used for testing, and the straight-line distance error of the two previous calculations is about 10-69 meters. Five sets of actual data from Google maps were used for the test, and the straight-line distance error of the fire point was about 30-79 meters. [14]

\section{Algorithm Principle}

The basic idea of single point positioning is to regard the optical axis of the monocular camera on the watchtower as the ray extending along the center of the field of vision. When the fire point is in the center of the image (the default position of the main image point), the first intersection point between the ray and the mountain is the fire point that needs to be positioned. [5]

The first step of the algorithm in this paper is to calculate the actual coordinate position when the fire image is at the center. The second step is to calculate the actual coordinate position when the fire point is at any pixel of the image.

\subsection{The Fire Point Is Centered in an Image}

In single point positioning, the angle between the camera optical axis and the horizontal plane is $\beta$, that is, the current pitch angle of the digital cloud console (camera optical axis). The angle between the horizontal projection of the camera axis and the $\mathrm{Y}$ axis (due north) is $\alpha$, that is, the current rotation angle of the digital cloud console. See figure 1.

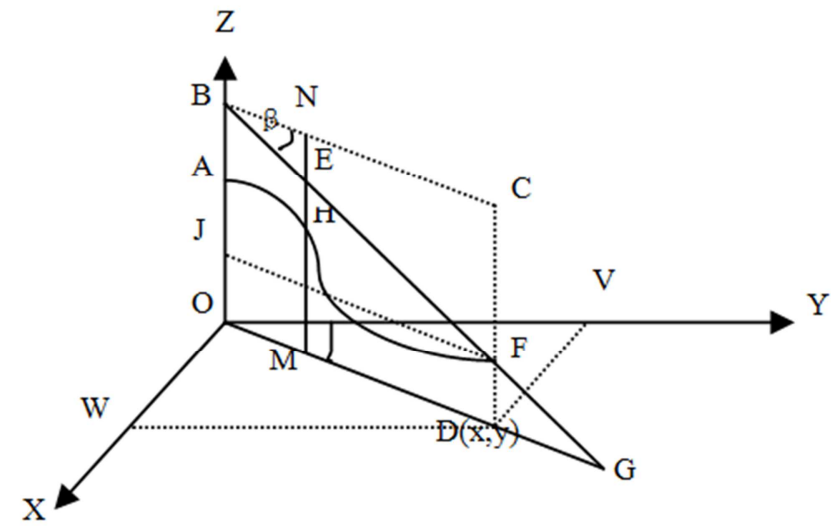

Figure 1. The coordinates of the fire point are calculated according to the rotation angle and pitch angle.

If the fire point is at the center of the image, the rotation angle and pitch angle of the fire point are the rotation angle and pitch angle of the camera optical axis. The following algorithm is given the rotation angle and pitch angle of the camera to solve the geographic coordinates of the fire point, namely the longitude and latitude of the fire point. [15]

First, assuming that the geodetic coordinates and rectangular coordinates are overlapped, the normal north direction (OY axis) is demarcated as the initial zero angle of the digital cloud console, and the direction of fire occurrence is determined by the rotation angle of the cloud console. Secondly, the specific position of the fire point is determined by the pitch angle. The mathematical model was established as shown in figure 1. Line $\mathrm{AB}$ is the height of the observation tower, OA is the height of the mountain, $\mathrm{F}$ is the imaginary fire point, and $\mathrm{D}$ is the projection of point $\mathrm{F}$ on the $\mathrm{x}-\mathrm{y}$ plane of the coordinate system. By solving the length of OD in the line segment, the projection distance of the fire point from the observation tower can be obtained, and the longitude and latitude information of the fire point can be obtained.

In order to speed up the operation, the system uses the method of combination of progressive exhaustion and dichotomy to solve the coordinates of point F. The specific orientation of the fire point can be determined by rotation angle $\alpha$, and the horizontal distance from the fire point to the cloud console can be determined by elevation angle $\beta$. The plane composed of points $\mathrm{O}, \mathrm{B}, \mathrm{C}$ and $\mathrm{D}$ is shown in figure 1 . In this system, the front-end video monitoring infrared camera is a fixed-focus lens with a visual range of $10 \mathrm{~km}$. According to the practical application, the positioning algorithm adopted in this paper starts from the origin $\mathrm{O}$ (that is, the projection of the observation tower on the ground, XOY overlaps with the sea level) and takes a point $m$ on ray OG every 1000 meters (step length) to find the sum of ground elevation $\mathrm{MH}$ at point $\mathrm{M}$ on the ray and angle $\beta$ opposite edge EN. Then subtract the sum from the height of the watchtower $\mathrm{OB}$ (equal to $\mathrm{MN}$ ), to get the value of $\mathrm{EH}$. It can be seen that as point $\mathrm{M}$ on ray $\mathrm{OG}$ is continuously evaluated every 1000 meters in front, the value of EH will 
gradually approach to zero (because terrain changes irregularly, elevation does not necessarily approach to zero in one direction). When the calculated EH value is less than zero, it means that the selected point $M$ exceeds the projection point $\mathrm{D}$ of the fire point $\mathrm{F}$ on $\mathrm{OG}$. At this time, go back to the previous selected point and continue to take the point every 100 meters until the calculated $\mathrm{EH}$ value is less than zero again. At this time, the error of OM value obtained is within 100 meters. In order to be more accurate, the binary method is used to determine the fire point $\mathrm{D}$. Then solve the coordinates corresponding to point D:

$$
\begin{aligned}
& x=O D * \sin (\alpha) \\
& y=O D * \cos (\alpha)
\end{aligned}
$$

Finally, based on the latitude and longitude of point $\mathrm{O}$ and $\mathrm{D}(\mathrm{x}, \mathrm{y})$ coordinates, it is easy to calculate the longitude and latitude coordinates of point D. As for the value of each elevation $\mathrm{MH}$, whose point $\mathrm{M}$ moves forward gradually through until it is close to point $\mathrm{D}$, can be obtained conveniently from the obtained rectangular coordinate position of point $\mathrm{M}$, and the corresponding longitude and latitude of the point which can be calculated according to the longitude and latitude of point $\mathrm{O}$.

\subsection{The Fire Point Image Is Located at Any Pixel Point}

B

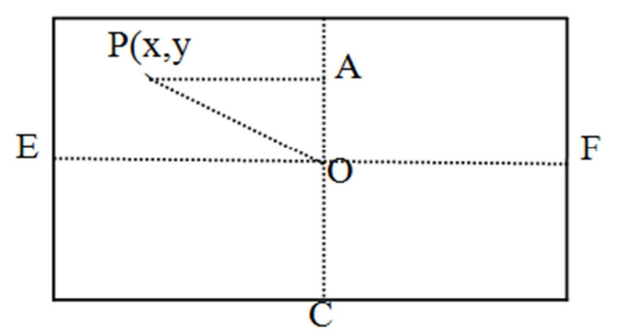

Figure 2. The fire point image $P(x, y)$ is located at any position of pixel coordinates.

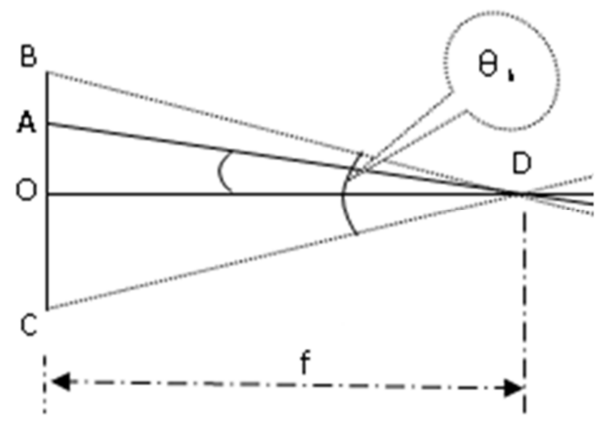

Figure 3. Principle of fixed-focus camera imaging.

If the latitude and longitude calculation method of the above fire point at the center of the image is available, the following procedure is to find out the angle difference between the rotation angle and pitching angle of the camera that needs to be adjusted when the fire point is at any point in the image.

As shown in figure 2, a mapping is established between the plane pixel coordinates and the three-dimensional spatial coordinates. The actual width of the image is $w \mathrm{~mm}$, and the pixel coordinates are $w_{x}$ pixels. The actual height of the image is $\mathrm{h} \mathrm{mm}$ and the pixel coordinates are $h_{x}$ pixels. The top left corner of the image is the zero coordinate position, the horizontal direction is the $\mathrm{x}$ pixel coordinate, and the vertical direction is the y pixel coordinate. Suppose the image of the fire point is at the pixel point $\mathrm{P}(\mathrm{x}, \mathrm{y})$. The camera's horizontal field of view angle is $\theta_{\mathrm{w}}$, its vertical field angle is $\theta_{\mathrm{h}}$, and the focal length of the fixed-focus camera is $\mathrm{f} \mathrm{mm}$. As shown in figure 3 , according to the imaging principle of the fixed-focus camera, in the BOD of right triangle, the actual height of the image in $\mathrm{mm}$ can be calculated:

$$
\begin{array}{r}
\mathrm{OB}=\mathrm{OD} * \tan \left(\theta_{\mathrm{h}} / 2\right) \\
\mathrm{h} / 2=\mathrm{f} * \tan \left(\theta_{\mathrm{h}} / 2\right) \\
\mathrm{h}=2 * \mathrm{f} * \tan \left(\theta_{\mathrm{h}} / 2\right)
\end{array}
$$

Then, the actual (length $\mathrm{mm}$ ) ratio of $\mathrm{OA} / \mathrm{OB}$ in figure 3 is equal to their pixel ratios:

$$
\begin{gathered}
\text { OA/OB }=\left(\mathrm{h}_{\mathrm{x}} / 2-\mathrm{y}\right) /\left(\mathrm{h}_{\mathrm{x}} / 2\right) \\
\text { OA } /(\mathrm{h} / 2)=\left(\mathrm{h}_{\mathrm{x}}-2 * \mathrm{y}\right) / \mathrm{h}_{\mathrm{x}} \\
\mathrm{OA}=\mathrm{h} *\left(\mathrm{~h}_{\mathrm{x}}-2 * \mathrm{y}\right) /\left(2 * \mathrm{~h}_{\mathrm{x}}\right)
\end{gathered}
$$

Calculate and get the actual distance of OA.

So, if you want to adjust the camera optical axis along the vertical direction to point to the same level, need to adjust the angle for: $\Delta \mathrm{y}=\angle \mathrm{ODA}$, can be calculated as follows. In right triangle $\mathrm{AOD}$ :

$$
\begin{gathered}
\tan \left(\Delta_{\mathrm{y}}\right)=\mathrm{OA} / \mathrm{OD} \\
\tan \left(\Delta_{\mathrm{y}}\right)=\mathrm{h} *\left(\mathrm{~h}_{\mathrm{x}}-2 * \mathrm{y}\right) /\left(2 * \mathrm{f} * \mathrm{~h}_{\mathrm{x}}\right) / \mathrm{f}=\mathrm{h} *\left(\mathrm{~h}_{\mathrm{x}}-\right. \\
2 * \mathrm{y}) /\left(2 * \mathrm{f}^{2} * \mathrm{~h}_{\mathrm{x}}\right)
\end{gathered}
$$

Then by calculating numerical inverse trigonometric function, angle $\Delta_{y}$ can obtain. $\Delta_{y}$ is the number which Fire needs to adjust the angle of the vertical. If the point, as we assume that, is in the center of the upper left is, the fire pitching angle should be adjusted to: $\beta-\Delta_{y}$. If the fire point is located at the upper right, lower left and lower right of the image center, the corresponding processing can be done. The difference is that when the fire point is above the horizontal line EF (Figure 2), the adjustment angle is reduced. And point below the image center horizontal line EF, the adjustment angle is increased, that is $\beta+\Delta_{y}$.

Similarly, the number of angles from the camera optical axis to point $\mathrm{P}$ in the horizontal direction can be obtained as:

$$
\tan (\Phi)=\mathrm{w} *\left(\mathrm{w}_{\mathrm{x}}-2 * \mathrm{x}\right) /\left(2 * \mathrm{f}^{2} * \mathrm{w}_{\mathrm{x}}\right)
$$

Here $\Phi$ not $\Delta_{x}$ is used to express point of view, because here $\Phi$ is not the angle which horizontal rotation angle need to adjust. It's angle $\angle \mathrm{CBF}$ in figure 4 . If you want to get the angle of $\angle \mathrm{MOY}=\Delta_{x}$ which horizontal rotation angle need to adjust, you need to do some operations. 


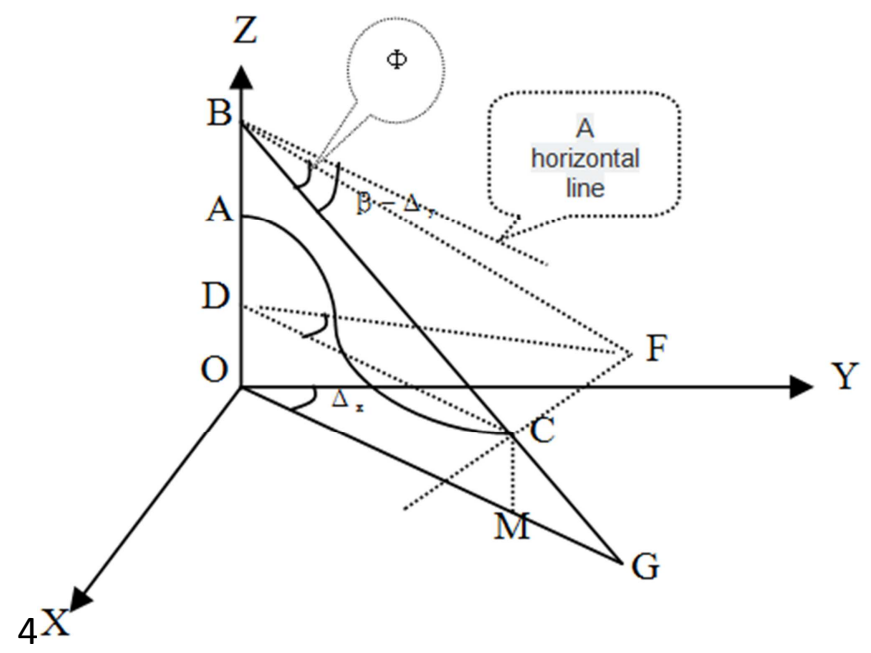

Figure 4. The fire point is adjusted vertically to the horizontal line in the center of the image.

According to the above analysis, if $\mathrm{CD} / / \mathrm{OM}, \mathrm{F}$ is the actual fire point, then $\mathrm{FC} \perp \mathrm{CD} \mathrm{FC} \perp \mathrm{CD}$, that is, FC perpendicular to any line segment through $\mathrm{C}$, that is, $\mathrm{FC}$ perpendicular to the plane $\mathrm{BCD}$. It can be seen that angle $\angle \mathrm{BCD}$ is equal to the adjusted pitch angle:

$$
\angle \mathrm{BCD}=\beta-\Delta_{\mathrm{y}}
$$

Therefore, according to 2.1, we can calculate the distance $\mathrm{OM}$ between point $\mathrm{M}$ that is projection of point $\mathrm{C}$ and point $\mathrm{O}$, as well as the height $\mathrm{CM}$ at point $\mathrm{C}$. In right triangle $\mathrm{BCD}$ :

$$
\begin{gathered}
\mathrm{CD}=\mathrm{OM} \\
\mathrm{BC}=\mathrm{CD} / \cos \left(\beta-\Delta_{\mathrm{y}}\right)
\end{gathered}
$$

In right triangle $\mathrm{BCF}$,

$$
\mathrm{CF}=\mathrm{BC} * \tan (\Phi)=\mathrm{CD} / \cos \left(\beta-\Delta_{\mathrm{y}}\right) * \tan (\Phi)
$$

In a right triangle $\mathrm{CDF}$,

$$
\begin{gathered}
\tan (\angle \mathrm{CDF})=\mathrm{CF} / \mathrm{CD}=\left(\mathrm{CD} / \cos \left(\beta-\Delta_{\mathrm{y}}\right) *\right. \\
\tan (\Phi)) / \mathrm{CD}=\tan (\Phi) / \cos \left(\beta-\Delta_{\mathrm{y}}\right) \\
\tan (\angle \mathrm{MOY})=\tan (\Phi) / \cos \left(\beta-\Delta_{\mathrm{y}}\right) \\
\tan (\Delta \mathrm{X})=\tan (\Phi) / \cos \left(\beta-\Delta_{\mathrm{y}}\right)
\end{gathered}
$$

To solve the inverse trigonometric function can get $\Delta_{X}$ degree.

Likewise, if the fire, as we assume that, is in the center of the upper left point, horizontal rotation angle should be adjusted as: $\alpha-\Delta_{X}$. As shown in figure 2 , if the fire point is located at the upper right, lower left and lower right of the image center point, corresponding processing can be done. The difference is that when the fire point is on the vertical line $\mathrm{BC}$ in the center of the image to the left, the adjustment angle is reduced. The point is located at the central vertical line $\mathrm{BC}$ to the right, the adjustment angle is increased, that is $\alpha+\Delta_{X}$.

At this point, the angle $\Delta_{x}$ and $\Delta_{y}$ which the fire point $\mathrm{P}$ need to adjust is evaluated. Then, according to the method of 2.1, the longitude and latitude values of the fire point image at any point in the image can be calculated.

Conversely, given the latitude and longitude of the fire point, calculating the horizontal rotation angle and the vertical pitch angle is much easier. As shown in figure 1 , no matter the fire point image is located in the center or other image positions, the distance of OD can be calculated by knowing the longitude and latitude of $\mathrm{O}$ and fire point $\mathrm{F}$, and the elevation DF of point F can be obtained by consulting the map data of longitude and latitude of F. FJ is parallel and equal to OD. In the right triangle BFJ,

$$
\begin{gathered}
\tan (\angle \mathrm{BFJ})=\mathrm{BJ} / \mathrm{FJ}=(\mathrm{OB}-\mathrm{OJ}) / \mathrm{OD}=(\mathrm{OB}-\mathrm{DF}) / \mathrm{OD}(19) \\
\tan (\beta)=(\mathrm{OB}-\mathrm{DF}) / \mathrm{OD}
\end{gathered}
$$

Then by the inverse trigonometric function to calculate the $\beta$, get the pitching angle.

The calculation process of rotation angle $\alpha$ is as follows: given that the longitudes and latitudes of $\mathrm{O}$ point and point $\mathrm{D}$, and their longitudes are equal to each other, and both are equal to the longitude of $\mathrm{O}$ point, namely the OY axis (due north), the coordinate length $\mathrm{OV}$ of point $\mathrm{D}$ on the $\mathrm{OY}$ axis can be calculated based on the known longitude and latitude of two points. Similarly, if two latitudes are equal, both are equal to the latitude of point $\mathrm{O}$, namely the $\mathrm{OX}$ axis, and the length $\mathrm{OW}$ and $\mathrm{OV}$ of point $\mathrm{D}$ in the $\mathrm{OX}$ axis can be calculated.

In the right triangle ODV,

$$
\tan (\angle \mathrm{DOV})=\tan (\alpha)=\mathrm{DV} / \mathrm{OV}=\mathrm{OW} / \mathrm{OV}
$$

And then we get $\alpha$ by the inverse trigonometric function. At this point, the algorithm is done.

\section{Algorithm Programming and Test Results}

Due to the strict requirements for program running time in practical projects, $\mathrm{VC}++2010$ was chosen to implement this algorithm. The following is the test result based on the data provided by party A:

Watchtower longitude: 117.421123, watchtower latitude: 26.910223.

Peak height: 498.5570373535156, tower height: 31.2 meters.

The calculation procedure of the algorithm is to calculate the longitude and latitude of the fire point coordinates with the rotation angle and pitching angle provided first, and then verify the rotation angle and pitching angle through the calculated longitude and latitude. The test results of selecting 10 groups of data are shown in table 1 . 
Table 1. Test results of 10 groups of data provided by party A.

\begin{tabular}{|c|c|c|c|c|c|c|c|}
\hline & elevation & $\begin{array}{l}\text { Longitude error } \\
\text { with party A's data }\end{array}$ & $\begin{array}{l}\text { Latitude error with } \\
\text { party A's data }\end{array}$ & $\begin{array}{l}\text { Rotation Angle error } \\
\text { with party A's data }\end{array}$ & $\begin{array}{l}\text { Pitch Angle error with } \\
\text { party A's data }\end{array}$ & pixel & $\begin{array}{l}\text { Two calculation } \\
\text { Distance error } \\
\text { (m) }\end{array}$ \\
\hline 69.08 & 270 & 0.00166844806 & 0.0078694835217 & -0.008270377865 & -0.0541829317498 & Image center & 69.08 \\
\hline 29.38 & 263 & -0.001023300857 & 0.0318863166278 & -0.004846496556 & -0.07633245093351 & Image center & 29.38 \\
\hline \multirow[t]{2}{*}{20.77} & 271 & -0.001612930703 & 0.0666358211569 & -0.004696567598 & -0.01322311369636 & Image center & 20.77 \\
\hline & \multicolumn{2}{|c|}{$\begin{array}{l}\text { The data elevation below is the } \\
\text { same }\end{array}$} & & \multicolumn{2}{|c|}{$\begin{array}{l}\text { Party A only gives two angles of the camera } \\
\text { optical axis and cannot calculate the difference. } \\
\text { So here's just the calculated angle of fire }\end{array}$} & \multicolumn{2}{|c|}{$\begin{array}{l}\text { The fire is not in the center of the } \\
\text { image }\end{array}$} \\
\hline 24.91 & 252 & -0.000798299876 & 0.045141260032 & 174.79673643097 & -5.5987999740956 & 161,125 & 24.91 \\
\hline 9.91 & 252 & -0.000798299876 & 0.0463243280346 & 174.761190270161 & -5.4442162007669 & 124,117 & 9.91 \\
\hline 51.06 & 252 & -0.001380059947 & 0.0463422364352 & 173.917173757836 & -5.44417427372226 & 175,80 & 51.06 \\
\hline 34.11 & 252 & -0.000537487895 & 0.0428366861022 & 175.350966425067 & -5.93581060434108 & 174,163 & 34.11 \\
\hline 46.24 & 252 & -0.00057649032 & 0.047398714806 & 174.502211548585 & -5.29790387718708 & 10,7 & 46.24 \\
\hline 46.60 & 252 & 0.001499521223 & 0.0432582020195 & 178.424079973893 & -5.93596647395889 & 7,240 & 46.60 \\
\hline 20.30 & 252 & 0.000456267861 & 0.0452345091851 & 176.263197963512 & -5.59887354801865 & 8,115 & 20.30 \\
\hline
\end{tabular}

Select the actual observation point near Qianling Mausoleum in Qian county, Shaanxi province on Google maps:

Watchtower longitude: 108.2025432587, watchtower latitude: 34.58679621171.

Peak height: $859.19 \mathrm{~m}$, tower height: $31.2 \mathrm{~m}$.

The algorithm operation process is that the rotation Angle and pitch Angle of the camera are calculated with the actual longitude and latitude coordinates first, and then the longitude and latitude coordinates are verified again through the calculated rotation Angle and pitch Angle. Select 5 groups of actual coordinate points, and the test results are shown in table 2.

Table 2. The test results of five sets of actual data on Google maps.

\begin{tabular}{|c|c|c|c|c|c|c|}
\hline $\begin{array}{l}\text { The actual } \\
\text { longitude }\end{array}$ & $\begin{array}{l}\text { The actual } \\
\text { latitude }\end{array}$ & $\begin{array}{l}\text { The calculated } \\
\text { rotation angle }\left({ }^{\circ}\right)\end{array}$ & $\begin{array}{l}\text { The calculated pitch } \\
\text { angle }\left({ }^{\circ}\right)\end{array}$ & Longitude error & Latitude error & $\begin{array}{l}\text { Error } \\
(\mathrm{m})\end{array}$ \\
\hline 108.2264471054 & 34.5201356281 & 131.095916524343 & -3.96994214131552 & -0.000473080541 & 0.0014055156404 & 71.8907 \\
\hline 108.2412099838 & 34.5152205662 & 120.08907936983 & -2.97246252147639 & -0.000205533168 & 0.0005281141079 & 29.362 \\
\hline 108.1980800629 & 34.5245200429 & 192.924547857655 & -5.71446555386903 & 0.000159132852 & 0.0022051496912 & 78.6826 \\
\hline 108.2439136505 & 34.5662311502 & 98.8455509364967 & -3.40706558345682 & -0.000357962541 & 0.0002234812578 & 40.6022 \\
\hline 108.2424545288 & 34.4963709147 & 125.345741338131 & -2.87963541072828 & -0.000398474322 & 0.0010962396525 & 58.5403 \\
\hline
\end{tabular}

It should be noted that all test data is the same result of repeated calculation, only the calculation time slightly changed.

\section{Conclusion}

In this paper, forest fire automatic positioning algorithm is researched by the use of camera calibration technology and three-dimensional space analysis on the digital video monitoring system. By means of DEM model, the location of fire point on topographic section is determined by exhaustive search and dichotomy. According to the characteristics of terrain change in forest region, the mapping between plane pixel coordinates and three-dimensional spatial coordinates is established by using the translation method of camera lens optical axis in space, which simplifies the algorithm and improves the calculation accuracy. The characteristic of the algorithm in this paper is to abandon the reference window frequently used in the original general algorithm, simplify the algorithm, reduce the complexity of the algorithm, reduce the intermediate calculation link, avoid the cumulative error of many calculations, improve the accuracy of calculation, and compress the program running time. In this paper, a total of $10+5$ groups of data were used for testing, and the calculated fire point position error range was 10-79 meters, with an average error of 42.10 meters. The practical application shows that this localization algorithm can satisfy the automatic localization of forest fire point and is an important part of the intelligent forest monitoring system.

\section{References}

[1] Zhuang Zhemin, Hailong Liao, et al. Study on the Location of Early Fire Source in Inclined Wind Field Based on Weighted Distance Difference Method. Journal of Safety and Environment, 2011, vol. 11 (3): pp. 177-181

[2] Wang S, BERENTSEN M, and KAISER T. Signal Processing for Fire Location Using Temperature Sensor Arrays. Fire Safety Journal, 2005, vol. 40 (8): pp. 689-697

[3] Zhemin Zhuang, XinFeng Zhang, Kalin Li, et al. Method Research Fire Source Locaion Based on Planar Circluar Sensor Arrays. Chinese Journal of Sensors and Actuators. 2009, vol. 22 (8): pp. 1208-1212

[4] Guangqun Yang, Ning Han. Study on Forest Fire Location Method Based on Camera Calibration Technology. Journal of Safety and Environment, 2013, vol. 13 (1): pp. 215-219.

[5] Zhemin Zhuang, Hailong Liao, Shengqiang Huang, et al. On the Early Fire Source Locating Method Base on the Weighted Differential Distance Approach in the Skew Wind Field. Journal of Safety and Environment, 2011, vol. 11 (3): pp. 177-181.

[6] Maolin Qiu, Songde Ma, Yi Li. Overview of Camera Calibration for Computer Vision. Acta Automatica Sinica, 2000, vol. 26 (11): pp. 43-55 
[7] Lifan Fei. Establishment of Digital Ground Model (DTM) and Its Application in Agricultural Planning. Regional Research and Development, 1988, 7 (4): pp. 42-43

[8] Jian Zhang, Guangqun Yang, Ning Han GIS- Based Positioning Methods in Video Monitoring of Forest Fires. Forestry Machinery \& Woodworking Equipment, 2009, vol. 36 (5): pp. 24-26.

[9] Shouyi Lu, Xiaoming Tang, Shengguo Wang. A Tutorial on the Use of Geographic Information Systems. China Forestry Press. Beijing, China, 1998. p152.

[10] Jianmin Yin. Research on Satellite Remote Sensing Fire Location Algorithm under ArcInfo. Nanjing Meteorological Journal, 2004, vol. 27 (5): pp. 688- 694.

[11] Survey Adjustment Group, School of Surveying and Mapping, Wuhan University. Error Theory and Basis of Measurement Adjustment. Wuhan: Wuhan University Press, 2003. pp. 207.
[12] Andrei B. Utkin, Armando Fernandes, Fernando Simoes, Alesander Lavrov and Rui Vilar. Feasibility of Forest - Fire Smoke Detection Using Lidar. International Journal of Wildland Fire, 2003, (12): pp. 159- 166.

[13] Morsdorf, F., Meier, E., K\#tz, B., Itten, K. I., Dobbertin, M., Allg\$wer, B. LIDAR - Based Geometric Reconstruction of Boreal Type Forest Stands at Single Tree Level for Forest and Wildland Fire Management. Remote Sensing of Environment, 2004, 92 (3): pp. 353- 362.

[14] Jian Zhang, Guangqun Yang, Ning Han, Yi Liang. Research on Forest Fire Location Algorithm Based on Video Monitoring System. Journal of Safety and Environment, 2009, Beijing, China, vol. 9 (1): pp. 127-130.

[15] Qifeng Yu, Yang Shang. Principle and Application Research of Photogrammetry. Science Press, Beijing, China, 2009: pp. 2325. 